\title{
Differential osteogenic activity of osteoprogenitor cells on HA and TCP/HA scaffold of tissue engineered bone.
}

\begin{abstract}
Biomaterial, an essential component of tissue engineering, serves as a scaffold for cell attachment, proliferation, and differentiation; provides the three dimensional (3D) structure and, in some applications, the mechanical strength required for the engineered tissue. Both synthetic and naturally occurring calcium phosphate based biomaterial have been used as bone fillers or bone extenders in orthopedic and reconstructive surgeries. This study aims to evaluate two popular calcium phosphate based biomaterial i.e., hydroxyapatite (HA) and tricalcium phosphate/hydroxyapatite (TCP/HA) granules as scaffold materials in bone tissue engineering. In our strategy for constructing tissue engineered bone, human osteoprogenitor cells derived from periosteum were incorporated with human plasma-derived fibrin and seeded onto $\mathrm{HA}$ or TCP/HA forming 3D tissue constructs and further maintained in osteogenic medium for 4 weeks to induce osteogenic differentiation. Constructs were subsequently implanted intramuscularly in nude mice for 8 weeks after which mice were euthanized and constructs harvested for evaluation. The differential cell response to the biomaterial (HA or TCP/HA) adopted as scaffold was illustrated by the histology of undecalcified constructs and evaluation using SEM and TEM. Both HA and TCP/HA constructs showed evidence of cell proliferation, calcium deposition, and collagen bundle formation albeit lesser in the former. Our findings demonstrated that TCP/HA is superior between the two in early bone formation and hence is the scaffold material of choice in bone tissue engineering.
\end{abstract}

Keyword: Bone tissue engineering; Fibrin; Hydroxyapatite; Periosteum; Tricalcium phosphate. 